\title{
A new model for production of fast light clusters in spallation reactions
}

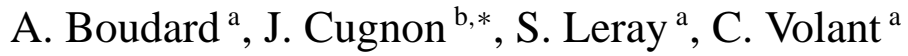 \\ a DAPNIA/SPhN, CEA/Saclay, F-91191 Gif-sur-Yvette cedex, France \\ b Université de Liège, Institut de Physique B5, Sart Tilman, B-4000 Liege 1, Belgium \\ Received 1 March 2004; received in revised form 5 April 2004; accepted 6 May 2004
}

Available online 20 May 2004

\begin{abstract}
A model is proposed to describe emission of light charged clusters during the cascade stage in nucleon-induced spallation reactions. It consists in implementing a surface percolation procedure into the Liège intranuclear cascade (INCL4) model: when a nucleon is ready to leave the nuclear surface, it is allowed to drag along a cluster of nucleons, under some conditions of closeness in phase space. This possibility relies on the instantaneous dynamical phase space occupancy in the nuclear surface. The following clusters are considered: $d, t,{ }^{3} \mathrm{He},{ }^{4} \mathrm{He}$. Good agreement is obtained with experimental data relative to heavy and medium-heavy targets at two different energies. It is shown that the implementation of light cluster emission in the cascade stage also improves our previous results for residue mass spectra.

(c) 2004 Elsevier B.V. All rights reserved.
\end{abstract}

PACS: 25.40.-h; 25.40.Sc; 25.45.-z; 24.10.Lx; 25.10.+s

Keywords: Spallation reactions; Intranuclear cascade model; Light cluster production

\section{Introduction}

There is a renewed interest in nucleon-induced spallation reactions in the GeV range, motivated especially, but not only, by various projects of spallation sources for acceleratordriven systems (ADS) or other applications (see for instance Ref. [1]). Most noticeably, spallation reactions lead to a copious emission of neutrons, accompanied by fewer protons

\footnotetext{
* Corresponding author.

E-mail address: cugnon@plasma.theo.phys.ulg.ac.be (J. Cugnon).
} 
and light nucleon clusters. Roughly speaking, the reaction process can be divided into two stages. The first one is dominated by fast particle emission and in the second one, the remnant of the target releases its remaining excitation energy by ordinary evaporation of slow particles (and/or by fission for heavy targets). Light charged clusters are emitted in the two stages, as suggested by the observation of their spectra [2-7]

The most successful model used to describe spallation reactions is the intranuclear cascade (INC) plus evaporation model. Recently we proposed an advanced version of the Liège intranuclear cascade model (INCL4) [8], which, coupled whith the K.-H. Schmidt evaporation-fission (ABLA) model $[9,10]$, gives a very accurate description of a large set of data (of different kinds) for proton and deuteron-induced reactions in the $200 \mathrm{MeV}$ to $2 \mathrm{GeV}$ range of incident energy per nucleon. The INCL4 model is basically a multiplescattering semi-classical model, which handles nucleon degrees of freedom explicitly. Therefore the model of Ref. [8] can accommodate emission of light clusters in the evaporation stage only. This deficiency is not a serious problem as far as global particle multiplicities are concerned. To fix the ideas, rough analyses of experimental data indicate that in a proton-induced reaction on a heavy target in the $\mathrm{GeV}$ range, the ratio of the number of nucleons appearing in the form of clusters emitted during the cascade stage to the total number of emitted nucleons, whatever their origin, lies between 5 and $10 \%$. However, the lack of cascade light cluster emission appears more serious in view of technological applications. Indeed light clusters correspond to gaseous elements $(\mathrm{H}, \mathrm{He})$, which are liable to create voids or other damages in materials. Therefore it is of utmost interest for the designers of (solid) spallation sources to have at their disposal a good model for the production of these elements. This motivates the present work in which we extent the INCL4 model in order to incorporate light cluster emission in the cascade stage.

We have however another even stronger motivation. For spallation reactions in the energy range under consideration, it is very hard to couple nucleon degrees of freedom and cluster degrees of freedom on a microscopic basis, i.e., to handle the formation of clusters from nucleons (and their possible destruction) via a microscopic and dynamical model involving the explicit effects of nuclear forces. Emission of light charged clusters prior to the eventual evaporation is generally described on a phenomenological basis, either by the standard coalescence model [11,12] (in momentum space) or by percolation models applied at the end of the cascade stage [13]. When a pre-equilibrium module is introduced between the cascade and evaporation stages [14,15], cluster production during this stage can be accounted for as follows: the nucleus is continuously described as an excited Fermi gas, but a parametrized probability for light cluster emission is attached to each "exciton" configuration. The parameters are usually determined by the study of light cluster cross-sections in low energy reactions, basically below $\sim 100 \mathrm{MeV}$. In heavy-ion physics, where more sophisticated models, such as QMD [16], BUU [17] and BNV [18] models are used, only nucleon degrees of freedom are taken into account in the cascade stage (before the freeze-out), although aggregation of nucleons may be observed during this stage. In practically all models, cluster emission is introduced through a local chemically equilibrated model $[19,20]$, or through a percolation procedure at the freeze-out followed by an after-burner (evaporation) step. Let us notice however that there exist even more sophisticated methods, such as those introduced by Feldmeier [21] and Horiuchi [22], which attempt to describe the effects of collisions on Slater determinants, modeling the 
state of the colliding system. In such approaches, clusters naturally arise at the end of the process, although some simplifying assumptions (regarding the wave function) have to be introduced. All models used in the heavy-ion case, giving moderate to good agreement for high energy cluster fragments, are however very time-consuming and have rarely been modified for nucleon-induced reactions. In the latter case the rapidity of the numerical models is crucial for applications.

We had also a third motivation. It was important for us to check whether the good agreement obtained in Ref. [8] is not too much modified when cluster emission in the cascade stage is taken into account.

In this paper, we want to present a model for light charged cluster production in nucleon-induced reactions. The model relies on the microscopic phase space occupancy at the nuclear surface. In our opinion, this is a reasonable physical hypothesis. Indeed, during nucleon-nucleus reactions the density of the target nucleus is only moderately perturbed [23] and theoretical investigations seem to indicate that deuterons do not really exist in ordinary nuclear matter at normal density [24-26]. A similar model has been proposed by Letourneau in Ref. [27] and embodies surface emission in the cascade from targets with a sharp surface. Other differences pertain to the construction of the clusters. Both this model and ours also have the advantage of allowing emission of clusters at any time during the cascade stage.

The paper is divided as follows. Section 2 contains a short reminder of the INCL4 model and presents the implemented cluster formation model. Section 3 shows typical results concerning cluster double differential cross-sections, cluster multiplicities and the effect of cluster formation on other observables. Finally our conclusion can be found in Section 4.

\section{The model}

The INCL4 model is described in detail in Ref. [8]. We just recall here the most relevant features for our purpose. It is a time-like INC model, which follows the fate of all particles as time develops. The particles travel in straight-line trajectories until two of them reach their minimum relative distance, in which case they can scatter, or until one of them reaches the surface, where it can be transmitted or reflected. At the beginning, the target nucleus is prepared according a Saxon-Woods density distribution $\rho(r)$ of radius $R_{0}$ and diffuseness parameter $a$, cut at $R_{\max }=R_{0}+8 a$, and to a uniform Fermi sphere momentum distribution with Fermi momentum $p_{F}$. However, position and momentum cannot generated independently, if the fact that fast nucleons can travel farther out than slow ones is to be taken into account. Actually, we take the momentum $\vec{p}$ of a nucleon at random in a sphere of radius $p_{F}$ and its position at random in a sphere of radius $R(p)$. The function $R(p)$, increasing with momentum $p$, is determined by requiring that the number of nucleons with momentum between $p$ and $p+d p$ is the same as the number of nucleons contributing to the slice of the density profile defined by

$$
\delta \rho(r)=\left(\rho(R(p))-\rho(R(p+d p)) \theta_{H}(R(p)-r),\right.
$$


where $\theta_{H}$ is the Heaviside function. In other words, the density profile can be viewed as divided in "horizontal" slices, defined by successive values of $R(p)$, and containing the same number of nucleons as the successive shells in the Fermi sphere, corresponding to equal intervals in $p$. Furthermore, nucleons are moving in a nuclear square potential well of constant depth $V_{0}$ and of radius $R(p)$. For $p>p_{F}$, the radius is taken as $R\left(p_{F}\right)=R_{\max }$. As shown in Ref. [8], this procedure ensures the stability of the target. More precisely, it allows us to conserve, on the average, the spatial and momentum distributions, if particles are propagated in the absence of collisions. The parameter $V_{0}$ can be chosen as to obtain a correct Fermi level energy. Finally, an improved statistical implementation of the Pauli blocking is applied and inelasticity is taken care of by introducing $\Delta$ and pion degrees of freedom.

Clusters can be emitted according to the following procedure:

(1) When a nucleon hits the surface and satisfies successfully the test for emission, i.e., has sufficient energy and escapes reflection (after the usual test of comparing a random number with the calculated transmission probability through the appropriate barrier including Coulomb potential for the protons), it is tested to see whether it belongs to a possible cluster. Such a cluster is defined as a set of nucleons which are sufficiently close to each other in phase space. Actually, the candidate cluster is constructed, starting from the considered nucleon, by finding a second, then a third, etc, nucleon fulfilling the following condition

$$
r_{i,[i-1]} p_{i,[i-1]} \leqslant h_{0}
$$

where $r_{i,[i-1]}$ and $p_{i,[i-1]}$ are the Jacobian coordinates of the $i$ th nucleon, i.e., the relative spatial and momentum coordinates of this nucleon with respect to the subgroup constituted of the first $[i-1]$ nucleons. The test on Jacobian coordinates has been preferred to a similar test on the relative coordinates $r_{i j}, p_{i j}$ for any pair $(i, j)$ of particles, because it precludes the appearance of clusters with exotic shapes (such as spaghetti's). In this work, the following light clusters are considered: $d, t,{ }^{3} \mathrm{He},{ }^{4} \mathrm{He}$.

(2) Fast nucleons are checked for emission at $R\left(p_{F}\right)$, in the outer fringes of the nucleus, where the density is very small and where they have little chance of being in a cluster. We are, however, forced to check particles for emission so far away, since they may undergo collisions before reaching this place. To correct for this, we move back the candidate for emission along its trajectory until it is at a distance $D$ outside the sphere of radius $R_{0}$, before building the possible clusters.

(3) We establish a hierarchy between clusters for testing their possible emission. It is evident, from the way clusters are constructed, that if the candidate nucleon belongs to a given cluster, say to an $\alpha$-particle, it also belongs to a lighter cluster. Clusters are checked for emission in the following priority list: ${ }^{4} \mathrm{He}>\left({ }^{3} \mathrm{He}\right.$ or $\left.t\right)>d$. In other words, the largest candidate cluster is first tested for emission. The total energy of the cluster, including the potential energy, and corrected by the binding energy of the cluster, should be positive in such a way that a composite with positive kinetic energy can be emitted. Furthermore, we check whether the cluster can tunnel through the appropriate Coulomb barrier, comparing a random number with the relevant transmission probability. If these conditions are met, the cluster is emitted in the direction of its c.m. momentum. Energy conservation is fulfilled in 
this process. If the test for emission fails, then the next cluster candidate in the priority list is checked for emission on the same criteria, and so on. If the test for emission fails for all clusters, the original candidate nucleon is emitted ( $\Delta$-resonances are not considered in the cluster formation).

This simple model appears as a kind of "surface-coalescence" model, compatible with two rather well established features: the small probability of having pre-existing clusters inside nuclei, at least in medium-heavy and heavy ones, and the necessary dynamical generation of correlated clusters of nucleons near the surface before emission. This is to be constrasted with the composite emission in pre-equilibrium models, in which a cluster can be emitted from an uncorrelated target with a suitable probability. Our approach presents also another appealing feature: clusters can be emitted at any time during the cascade stage. The present model presents some similarity with model proposed in Ref. [27]. Although the spirit is the same, they differ in the description of the surface (a sharp surface is used in Ref. [27]), in the construction of the clusters and in the hierarchy criterion for emission.

Our cluster production model utilizes the microscopic phase space distribution, as generated dynamically by the INC. It however contains some limited amount of phenomenology, since, as mentioned in the introduction, explicit coupling of individual nucleon and composite degrees of freedom is avoided and replaced by a geometrical construction, involving the introduction of the two parameters $h_{0}$ and $D$, whose values are given below.

\section{Results}

\subsection{Introduction}

We report here on our calculations and compare them with well documented data sets. We used our cascade code coupled with the evaporation code GEM [28] or with the KHSv3p version of the ABLA code [9,10], as in our previous work [8]. Although we are primarily interested in cascade emission, we nevertheless present the full spectra for light clusters. In KHSv3p, only evaporation of nucleons and alpha particles is considered, whereas GEM accommodates the evaporation of all light clusters.

In this first approach, aiming at demonstrating the potentialities of the model, we did not play with the parameters $h_{0}$ and $D$. Only a rough fit has been done with a single set of values, namely $h_{0}=387 \mathrm{MeV} \mathrm{fm} / c\left(=p_{F} \times 1.4 \mathrm{fm}\right)$ and $D=1.75 \mathrm{fm}$. The value of $h_{0}$ roughly corresponds to selecting a unit volume of phase space. The value of $D$ is such that the cluster is formed in a region of relatively low density on the average. Neglecting the improbable re-interaction (by strong interaction) with other nucleons on its way out, as it is implicitly assumed in our model, is therefore reasonable. The possibility of varying these parameters with excitation energy or other variables will be examined in a later work.

\subsection{Cluster double differential cross-sections}

Fig. 1 refers to the NESSI data [7] concerning the $p+$ Au reaction at $2.5 \mathrm{GeV}$, compared to our calculations with the ABLA evaporation code. An interesting aspect 

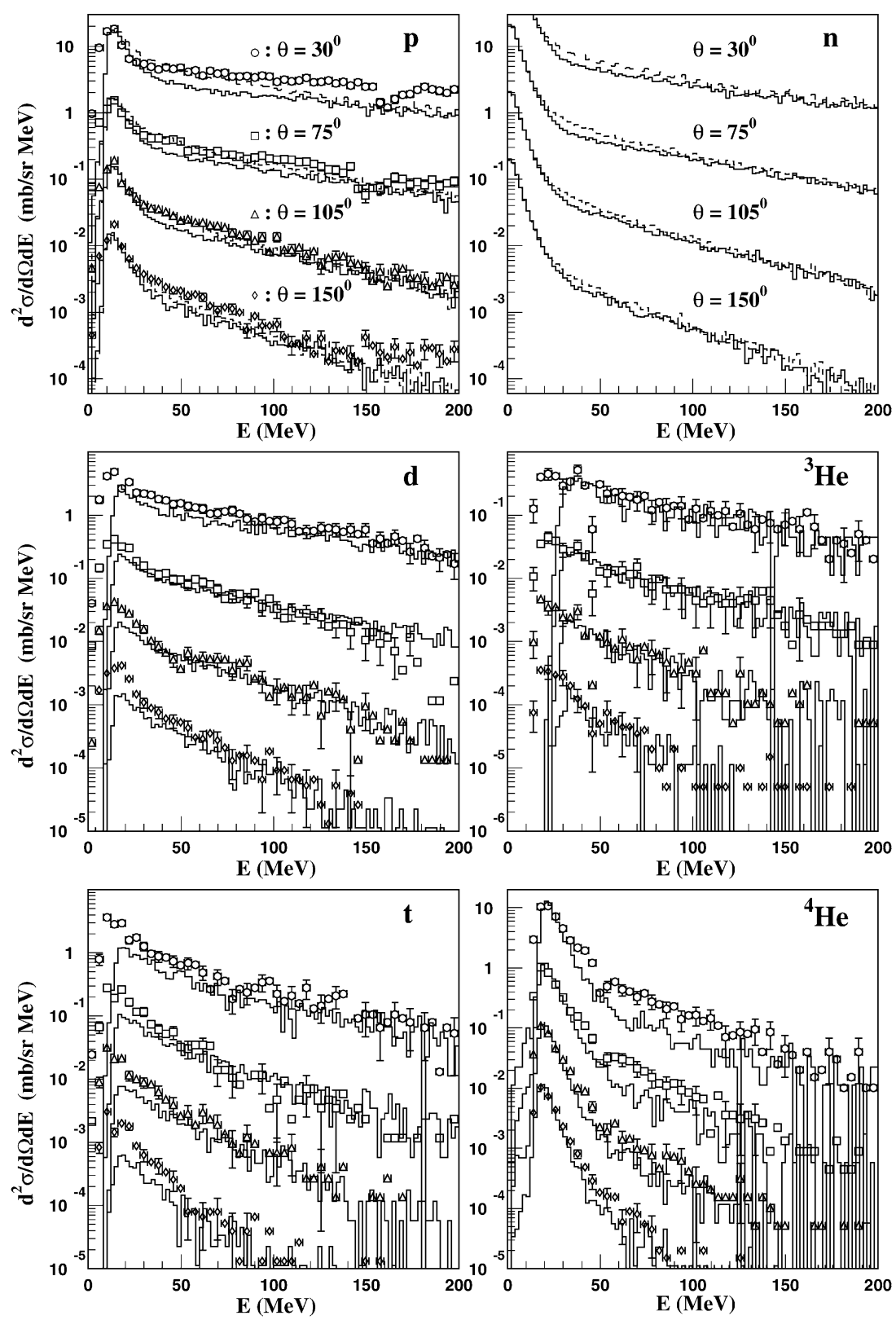

Fig. 1. Comparison of the results of our model, coupled to the ABLA evaporation code, for light charged cluster production in the $p+\mathrm{Au}$ system at $2.5 \mathrm{GeV}$ (histograms, full lines: with clusters, dotted lines: without clusters) with the experimental data (symbols) of Ref. [7]. The nature of the emitted particles is indicated in the panels. The different symbols correspond to different emission angles, which are given in the top panels. The predictions of the neutron cross-sections are given for the sake of comparison. In each panel, the cross-sections are given in absolute values for the smallest angle. They are multiplied by $10^{-1}, 10^{-2}$, etc., for the other angles, in increasing order. Note the different vertical scales. 
of these measurements is that particles are detected up to a kinetic energy of $200 \mathrm{MeV}$, allowing a meaningful test of cascade emission. As can be seen, the overall agreement is satisfactory. There are however some discrepancies. High energy protons $(E \gtrsim 30 \mathrm{MeV})$ are underestimated, especially at the most forward and most backward angles. Alphaparticle production in the cascade stage is also underestimated. Of course, the low energy part of the $d, t$ and ${ }^{3} \mathrm{He}$ spectra is missed, since the ABLA code does not accommodate evaporation of these particles. It is evident from Fig. 1 that the cascade production of these particles is not negligible. This will be quantified below when we discuss multiplicites. As expected, clusters in the cascade stage are formed at the expense of neutron and proton production (see the differences between the full and dashed histograms in the top panels). This is particularly noticeable for protons at small angles in the energy range spanning from 40 to $100 \mathrm{MeV}$, whose yield is clearly underestimated.

In Fig. 2, we display the same data and our results with the GEM evaporation code. The cascade spectra are the same, within statistical uncertainties (the simulation involves about 150.000 events). The differences bear only on the evaporation spectra. First, with the GEM code, the evaporation contributions are well described for deuterons and tritons. Second, the ${ }^{3} \mathrm{He}$ evaporation yield seems underestimated. Third, the proton evaporation cross-sections are slightly less satisfactory with GEM than with ABLA. In particular, the yield at the two largest angles is underestimated with GEM. Fourth, for ${ }^{4} \mathrm{He}$ evaporation, the peak height is well reproduced by the GEM model, but the slope of the decreasing part is steeper than in the ABLA model and steeper than the experimental slope.

In Fig. 3, we show the comparison of our predictions, using the same set of parameters as before, with the data of Ref. [6] for the $n+\mathrm{Bi}$ and $n+\mathrm{Cu}$ systems at $542 \mathrm{MeV}$. This time, we only show the results for one evaporation code (GEM), since the evaporation contributions are practically outside the range of the measurements. The agreement is again quite satisfactory but the trends have reversed, compared to the previous case: the proton yield is not underestimated any more and the $d$ and $t$ cluster cross-sections are somehow overestimated. It is nevertheless gratifying to see that with simple ingredients the gross features of the cascade spectra as functions of emission angle and energy are satisfactorily described, more especially they have been tested at two incident energies and for three targets.

We postpone the discussion of the properties of the formation mechanism to a forthcoming publication, but it is worth pointing out that, in the three systems mentioned above, light cluster production does not seem to correspond to a simple coalescence picture (in momentum space). Indeed, in this picture, and assuming the same shape for neutron and proton spectra, the cross-section for production of clusters of mass number $A_{c}$ is related to the proton cross-section by the relation [29]

$$
\frac{1}{\sqrt{E}} \frac{d \sigma}{d \Omega d E}(E)=C\left(\frac{1}{\sqrt{E_{p}}} \frac{d \sigma_{p}}{d \Omega d E_{p}}\left(E_{p}=E / A_{c}\right)\right)^{A_{c}},
$$

where $C$ is a constant, independent of $E$. The cascade part of the proton cross-section can be approximated by an exponential function:

$$
\frac{d \sigma_{p}}{d \Omega d E_{p}} \approx C^{\prime} \exp \left(-E_{p} / E_{0}\right)
$$



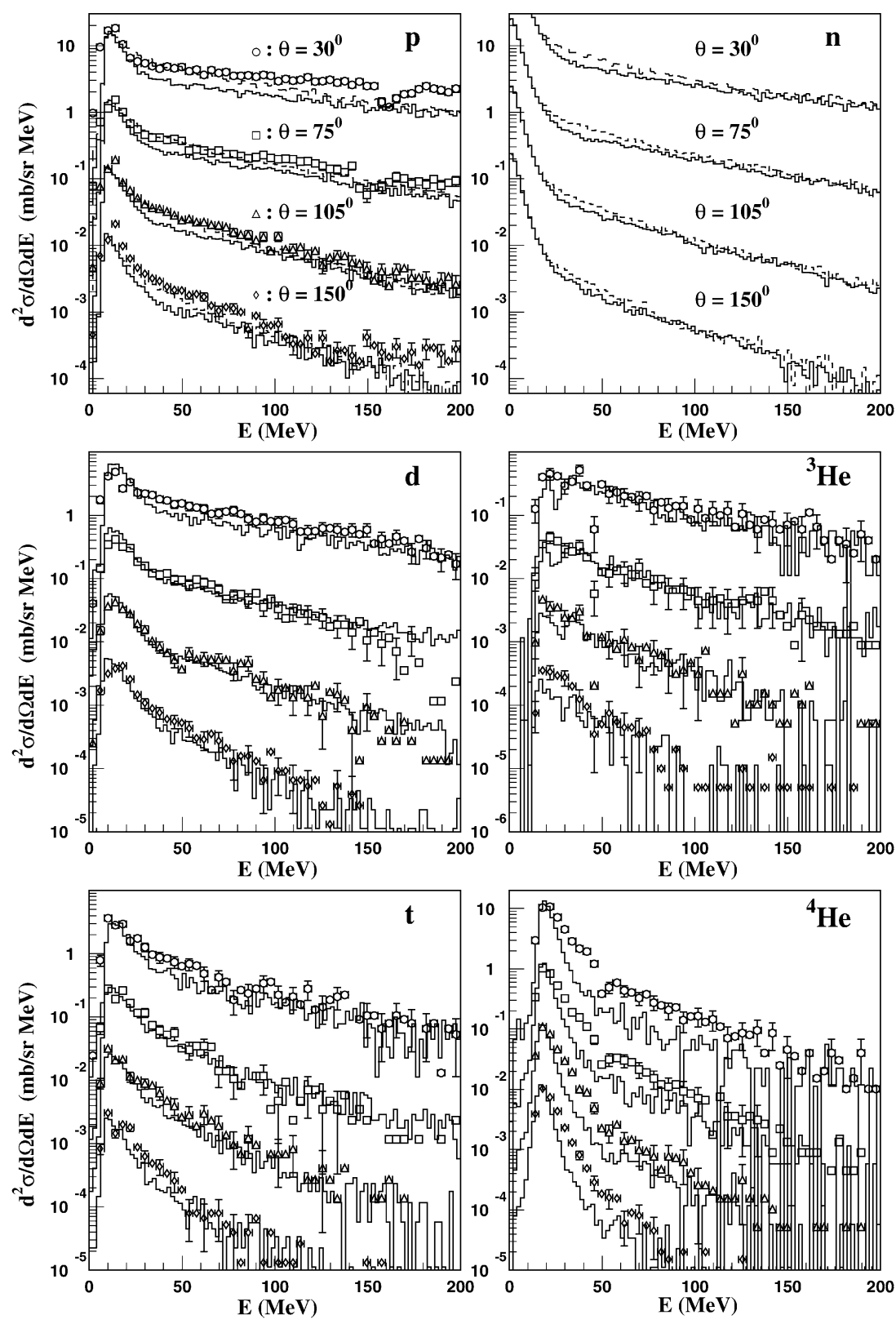

Fig. 2. Same as Fig. 1, but with the GEM evaporation code.

where $C^{\prime}$ is another constant independant of $E_{p}$. The cascade cluster cross-sections should then be approximately given by

$$
\frac{d \sigma}{d \Omega d E}(E) \approx C\left(C^{\prime} \sqrt{A_{c}}\right)^{A_{c}}(\sqrt{E})^{1-A_{c}} \exp \left(-E / E_{0}\right),
$$



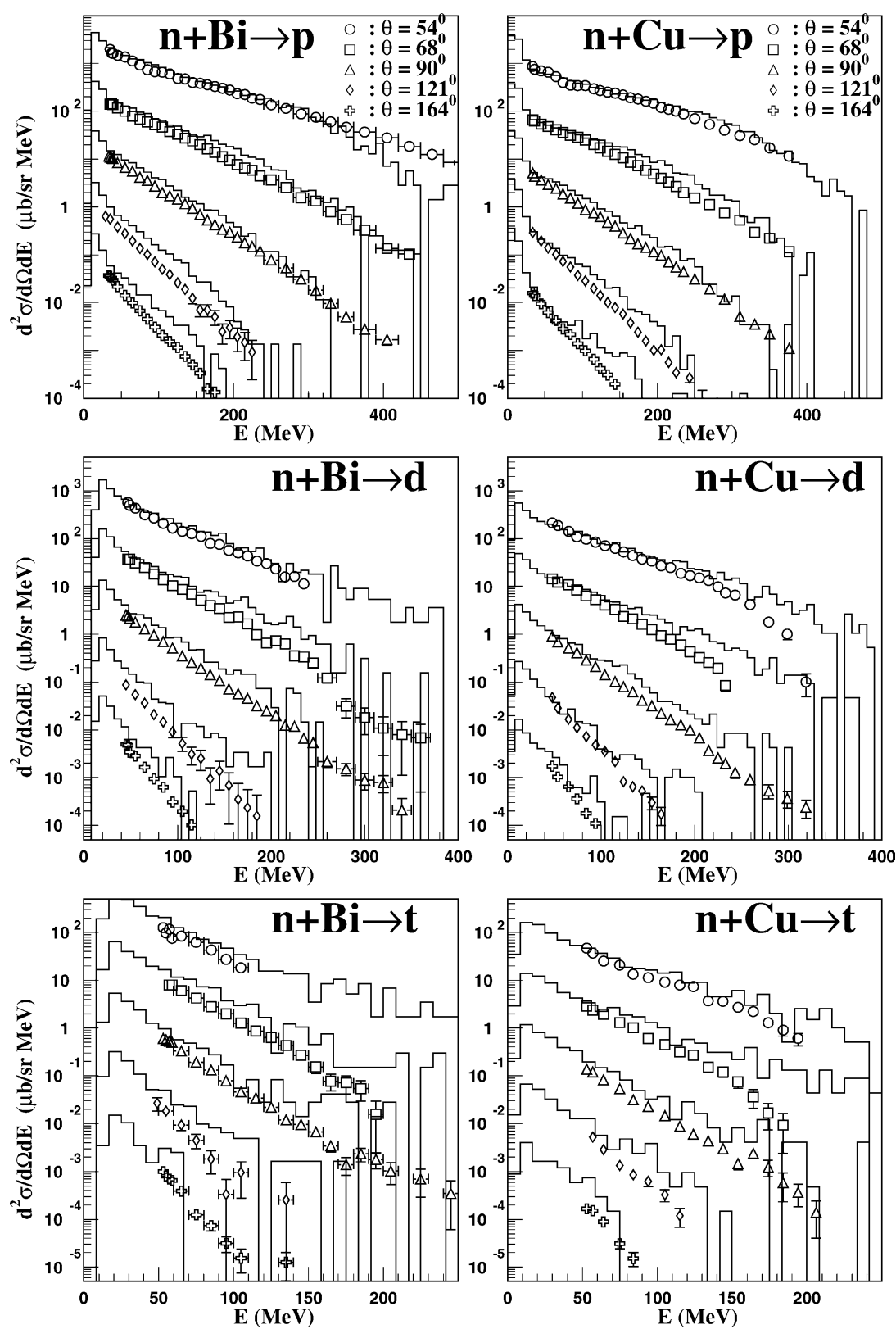

Fig. 3. Comparison of the results of our model (histograms) for double differential cross-sections in the $n+\mathrm{Bi}$ system (left panels) and in the $n+\mathrm{Cu}$ system (right panels) at $542 \mathrm{MeV}$, with the experimental data (symbols). The evaporation code GEM is used. The data are from of Ref. [6]. The different symbols correspond to different emission angles, which are given in the top panels. In each panel, the cross-sections are given in absolute values for the smallest angle. They are multiplied by $10^{-1}, 10^{-2}$, etc., for the other angles, in increasing order. Note the different vertical scales. 
i.e., they should basically display the same exponential decrease (the factor containing $\sqrt{E}$ is numerically unimportant here due to the limited range of energies; it corresponds to a slight hardening of the exponential decay). Obviously, this is not the case in Figs. 1 and 3 , both experimentally and in our model, demonstrating a more subtle mechanism. The quantity $E_{0}$ takes a larger value for deuterons and tritons than for protons, for the same emission angle. This situation is to be contrasted with the heavy-ion case where the simple coalescence model works rather well [30] in the same energy range. This was already acknowledged in Ref. [6].

\subsection{Particle multiplicities}

We now turn to particle multiplicities and compare the results with and without cluster formation in the cascade stage. The latter are given in Table 1. Several observations are in order. Let us concentrate first on the $p(2.5 \mathrm{GeV})+{ }^{197} \mathrm{Au}$ system. As expected, fewer free nucleons are emitted in the cascade stage, by about 20 percent. But this is overcompensated by the emission of nucleons within clusters. In the cascade stage, the total multiplicity of the emitted nucleons, free or bound, is increased by $\sim 10 \%$ for neutrons and $\sim 15 \%$ for protons. With the cluster emission scenario, the emission of nucleons is made easier for two reasons. First, removing a bound system costs less energy than removing all of its nucleons independently. Second, tunneling through the potential + Coulomb barrier favours emission of clusterized nucleons: for instance, the tunneling probability is smaller for an $\alpha$-particle than for a proton, but the test is applied only once for the latter. The probability of the uncorrelated emission of two protons and two neutrons is equal to the square of the proton emission probability multiplied by the square of the neutron emission probability. There is possibly a third reason, inherent to the scenario itself, which favours emission of a group of nucleons, that otherwise would have somehow diverging trajectories. The excitation energy at the end of the cascade is not really changed when the cluster emission is added. As a consequence, the evaporation multiplicities are not really changed either. Altogether, the total yield of emitted neutrons (either free or bound) is increased by $\sim 4 \%$ and that of emitted protons by $\sim 7 \%$. We also added in Table 1 the results for the $p+\mathrm{Pb}$ system at $1 \mathrm{GeV}$, with the GEM evaporation module, and at $1.2 \mathrm{GeV}$ with the KHSv3p evaporation module. The former case allows us to give a hint at the dependence of our results with the incident energy, using the (more complete) GEM evaporation model. The latter case provides with a meaningful comparison with our previous results (last column of Table 1 and Ref. [8]). It is interesting to note the reduction of the cascade neutron multiplicity by the introduction of cluster emission. The multiplicity of fast neutrons, with kinetic energy larger than $20 \mathrm{MeV}$, changes from 3.17 [8] to 2.69 when cluster emission is introduced and comes in slightly better agreement with the experimental value of $2.7 \pm 0.3$ [31]. Unfortunately, no other direct multiplicity measurement has been performed for the systems that we have investigated.

Let us comment on the results for particle multiplicities with cluster emission. Ratios of multiplicities are nearly the same for the two systems $p(2.5 \mathrm{GeV})+\mathrm{Au}$ and $p(1 \mathrm{GeV})+$ $\mathrm{Pb}$. Looking at our results with the GEM evaporation code, which are more complete, one finds that $\langle p\rangle /\langle n\rangle \approx 0.2,\langle d\rangle /\langle p\rangle \approx 0.3,\langle t\rangle /\langle d\rangle \approx 0.4,\left\langle{ }^{3} \mathrm{He}\right\rangle /\langle t\rangle \approx 0.25$ and $\langle\alpha\rangle /\langle p\rangle \approx$ 0.2 . It is interesting to note also that the ratio of the number of nucleons emitted inside 
Table 1

Comparison between particle multiplicities predicted by our model for three systems: $p(2.5 \mathrm{GeV})+{ }^{197} \mathrm{Au}$, $p(1 \mathrm{GeV})+{ }^{208} \mathrm{~Pb}$ and $p(1.2 \mathrm{GeV})+{ }^{208} \mathrm{~Pb}$. Multiplicities are splitted in INC and evaporation components. The evaporation model used in the calculations is indicated on the second line. The tildes refer to nucleons bound inside clusters. The excitation energy $E^{\star}$ at the end of the cascade stage (in $\mathrm{MeV}$ ) is also given

\begin{tabular}{|c|c|c|c|c|c|c|c|c|}
\hline & \multirow{2}{*}{\multicolumn{2}{|c|}{$\frac{p(2.5 \mathrm{GeV})+\mathrm{Au}}{\mathrm{ABLA}}$}} & \multirow{2}{*}{\multicolumn{2}{|c|}{$\frac{p(2.5 \mathrm{GeV})+\mathrm{Au}}{\mathrm{GEM}}$}} & \multirow{2}{*}{\multicolumn{2}{|c|}{$\frac{p(1 \mathrm{GeV})+{ }^{208} \mathrm{~Pb}}{\mathrm{GEM}}$}} & \multirow{2}{*}{\multicolumn{2}{|c|}{$\frac{p(1.2 \mathrm{GeV})+{ }^{208} \mathrm{~Pb}}{\mathrm{ABLA}}$}} \\
\hline & & & & & & & & \\
\hline & $\begin{array}{l}\text { without } \\
\text { clusters }\end{array}$ & $\begin{array}{l}\text { with } \\
\text { clusters }\end{array}$ & $\begin{array}{l}\text { without } \\
\text { clusters }\end{array}$ & $\begin{array}{l}\text { with } \\
\text { clusters }\end{array}$ & $\begin{array}{l}\text { without } \\
\text { clusters }\end{array}$ & $\begin{array}{l}\text { with } \\
\text { clusters }\end{array}$ & $\begin{array}{l}\text { without } \\
\text { clusters }\end{array}$ & $\begin{array}{l}\text { with } \\
\text { clusters }\end{array}$ \\
\hline$\langle n\rangle$ casc & 4.97 & 4.19 & 5.00 & 4.19 & 3.78 & 3.23 & 4.13 & 3.52 \\
\hline$\langle n\rangle$ evap & 10.56 & 10.68 & 10.39 & 10.54 & 9.66 & 9.82 & 10.58 & 10.75 \\
\hline$\langle p\rangle$ casc & 3.25 & 2.64 & 3.34 & 2.63 & 2.49 & 2.00 & 2.69 & 2.16 \\
\hline$\langle p\rangle$ evap & 1.09 & 1.08 & 0.93 & 0.91 & 0.35 & 0.35 & 0.54 & 0.54 \\
\hline$\langle\tilde{n}\rangle$ casc & - & 1.35 & - & 1.35 & - & 0.98 & - & 1.08 \\
\hline$\langle\tilde{n}\rangle$ evap & 1.78 & 1.74 & 2.41 & 2.38 & 1.41 & 1.39 & 1.22 & 1.21 \\
\hline$\langle\tilde{p}\rangle$ casc & - & 1.21 & - & 1.21 & - & 0.87 & - & 0.96 \\
\hline$\langle\tilde{p}\rangle$ evap & 1.78 & 1.74 & 2.25 & 2.21 & 1.32 & 1.30 & 1.22 & 1.21 \\
\hline$\langle n+\tilde{n}\rangle$ casc & 4.97 & 5.54 & 5.00 & 5.54 & 3.78 & 4.21 & 4.13 & 4.60 \\
\hline$\langle n+\tilde{n}\rangle$ evap & 12.34 & 12.41 & 12.80 & 12.92 & 11.07 & 11.21 & 11.80 & 11.96 \\
\hline$\langle p+\tilde{p}\rangle$ casc & 3.25 & 3.85 & 3.34 & 3.84 & 2.49 & 2.87 & 2.69 & 3.12 \\
\hline$\langle p+\tilde{p}\rangle$ evap & 2.87 & 2.82 & 3.17 & 3.12 & 1.67 & 1.64 & 1.76 & 1.745 \\
\hline$\langle n+\tilde{n}\rangle$ total & 17.31 & 17.95 & 17.80 & 18.46 & 14.85 & 15.42 & 15.93 & 16.55 \\
\hline$\langle p+\tilde{p}\rangle$ total & 6.22 & 6.66 & 6.51 & 6.96 & 4.16 & 4.51 & 4.45 & 4.86 \\
\hline$\langle d\rangle$ casc & - & 0.69 & - & 0.69 & - & 0.51 & - & 0.56 \\
\hline$\langle d\rangle$ evap & - & - & 0.42 & 0.42 & 0.16 & 0.165 & - & - \\
\hline$\langle t\rangle$ casc & - & 0.23 & - & 0.23 & - & 0.165 & - & 0.18 \\
\hline$\langle t\rangle$ evap & - & - & 0.19 & 0.20 & 0.10 & 0.10 & - & - \\
\hline$\left\langle{ }^{3} \mathrm{He}\right\rangle$ casc & - & 0.085 & - & 0.084 & - & 0.052 & - & 0.06 \\
\hline$\left\langle{ }^{3} \mathrm{He}\right\rangle$ evap & - & - & 0.023 & 0.022 & 0.005 & 0.0047 & - & - \\
\hline$\langle\alpha\rangle$ casc & - & 0.063 & - & 0.064 & - & 0.043 & - & 0.047 \\
\hline$\langle\alpha\rangle$ evap & 0.89 & 0.87 & 0.79 & 0.775 & 0.53 & 0.51 & 0.609 & 0.603 \\
\hline$\left\langle E^{\star}\right\rangle(\mathrm{MeV})$ & 179.2 & 180.2 & 179.2 & 180.6 & 135.9 & 137.5 & 148.5 & 150.6 \\
\hline
\end{tabular}

clusters to the total number of emitted nucleons (free or bound) amounts to $\sim 1 / 4$ in the two systems (actually 0.28 and 0.22 , respectively). The ratio of the number of nucleons emitted inside clusters in the cascade stage to the total number of nucleons emitted inside clusters lies around 0.4 . These figures grossly corroborate what is mentioned in the introduction. Let us however emphasize that the corresponding ratio between cascade yield to total yield varies strongly from cluster to cluster. For the $p(2.5 \mathrm{GeV})+\mathrm{Au}$ system, about $60 \%$ of the deuterons, $50 \%$ of the tritons and $80 \%$ of the ${ }^{3} \mathrm{He}$ clusters are emitted in the cascade stage. These values are slightly larger in the $p(1 \mathrm{GeV})+\mathrm{Pb}$ system. On the contrary, $\alpha$-particles are overwhelmingly emitted by evaporation ( $~ 92 \%$ for both systems). Let us finally mention that the theoretical ratios of multiplicities cited above exhibit the same trend as the experimental values reported in the Table 2 of Ref. [32], for $p+$ Au collisions 


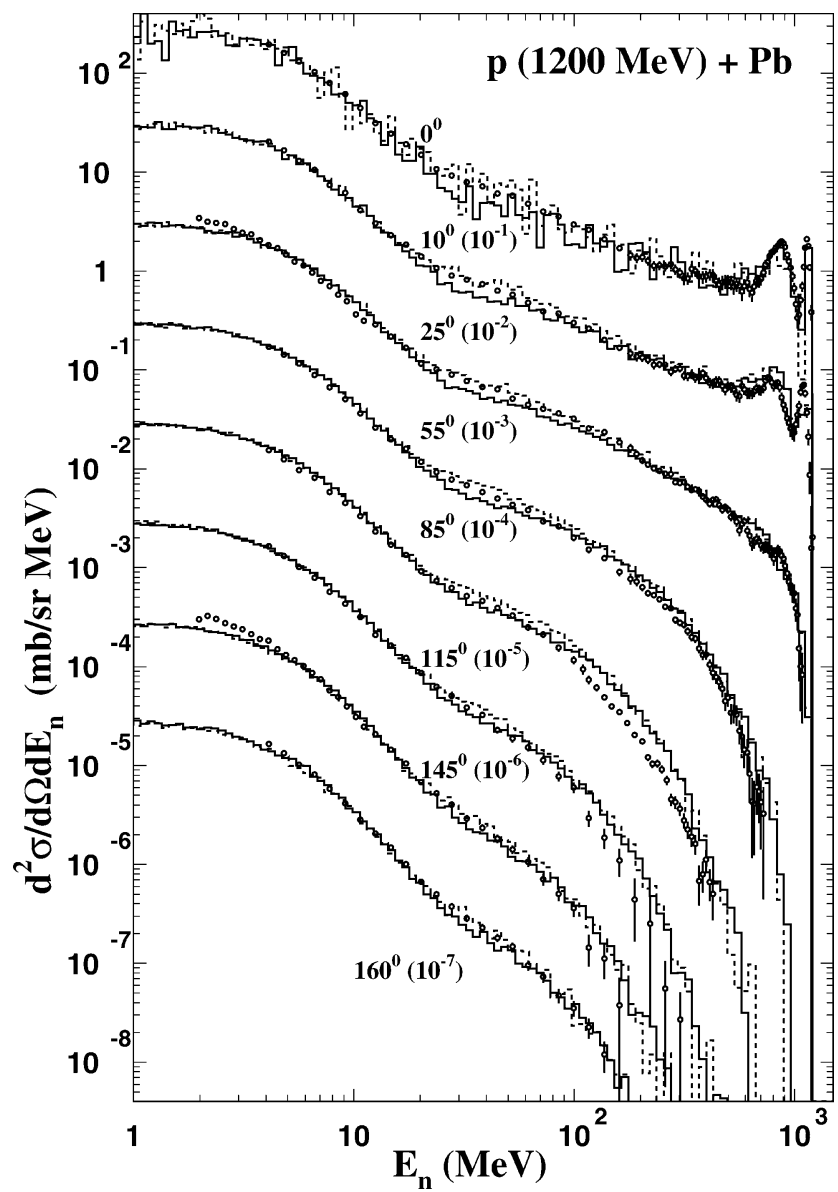

Fig. 4. Comparison of the results of our model (histograms) for neutron double differential cross-sections in $p+{ }^{208} \mathrm{~Pb}$ at $1.2 \mathrm{GeV}$, with (full lines) and without (dashed lines) clusters, with the experimental data (circles) of Ref. [31]. Cross-sections at $0^{\circ}$ are given in absolute values. For the other angles, they are multiplied by the indicated factors.

at $1.8 \mathrm{GeV}$. We did not attempt a quantitative comparison, mainly because the experimental cuts are not the same for all kinds of particles.

\subsection{Effects on other observables}

As indicated in Figs. 1 and 2, free neutron and free proton cross-sections are not diminished uniformly by the introduction of cascade cluster emission. The decrease is the more important for moderate energy nucleons (between 20 and $100 \mathrm{MeV}$ roughly). This is corroborated by Fig. 4, which displays the results for neutron double differential crosssection in $p+{ }^{208} \mathrm{~Pb}$ collisions at $1.2 \mathrm{GeV}$. Compared to our previous results [8], without clusters, the predictions are slightly poorer at 10 at $25^{\circ}$ and slightly better at $85^{\circ}$ and larger 


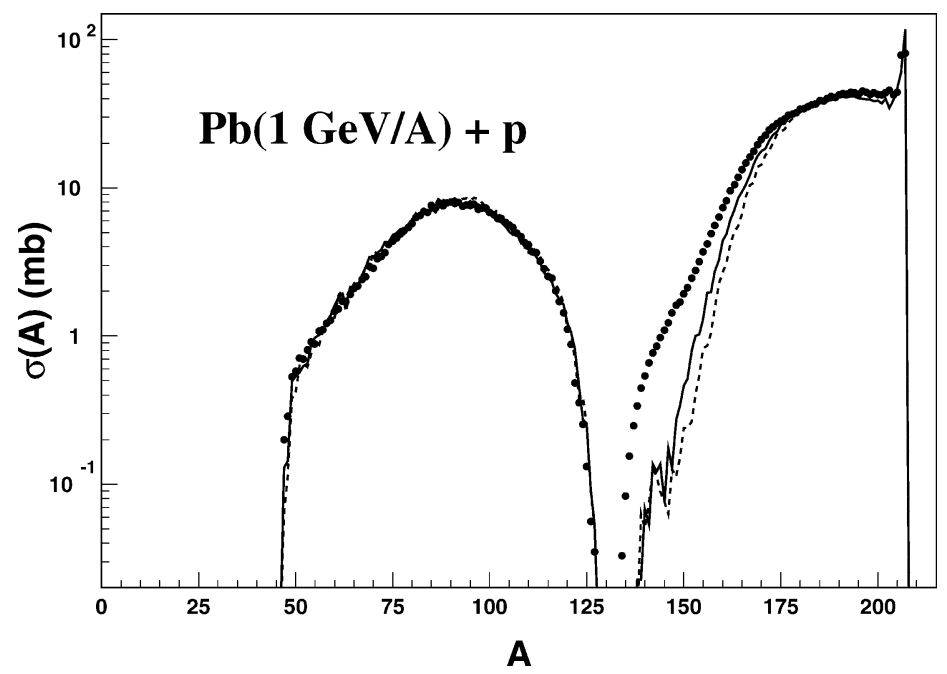

Fig. 5. Comparison of the results of our model (histograms) for residue mass production cross-section in the $p+{ }^{208} \mathrm{~Pb}$ system at $1 \mathrm{GeV}$, with (full line) and without (dashed line) clusters, with the experimental data (dots) of Ref. [33].

angles. Because the neutron yields are especially affected in the 20-50 MeV domain, the shapes of the spectra are somehow less satisfactory than before.

The introduction of cascade light cluster emission has another interesting and unexpected consequence. After the cascade stage, the remnant nucleus contains slightly less nucleons than before. Since the evaporation is not changed very much, the residues are slightly lighter (the small reduction of the excitation energy may also contribute to this effect). This is illustrated in Fig. 5, which shows the residue mass spectrum in the $p+\mathrm{Pb}$ system at $1 \mathrm{GeV}$. The lack of residue production in the low mass side of the fragmentation peak from which our previous calculation suffers [8] is partially reduced.

\subsection{Sizes of the clusters}

Let us close this section by discussing briefly the size of the "pre-clusters" appearing in our model, i.e., their extension in $r$-space and momentum space, at the moment of their construction, just before emission. In Fig. 6, we plot in the left panels the distribution of the distance $r$ separating the position of the nucleons from the center of mass, for the deuterons (upper part) and the alphas (lower part) constructed in our model. In the right panels, we display the distribution of $p$, the absolute value of the difference between the momentum of the nucleons and the total cluster momentum divided by its mass number, i.e., the absolute value of the momentum of the nucleons in the rest frame of the cluster. For deuterons, $r$ is half the relative distance and $p$ is the relative momentum. For large clusters, the distributions are just the nuclear densities in $r$ - and $p$-spaces multiplied by $r^{2}$ and $p^{2}$, respectively. The dashed lines in Fig. 6 give the same distributions for free clusters, as calculated with the Paris potential [34] for deuterons and with a Gaussian model with realistic parameters fitted on the experimental charge distribution for $\alpha$-particles. The "pre- 

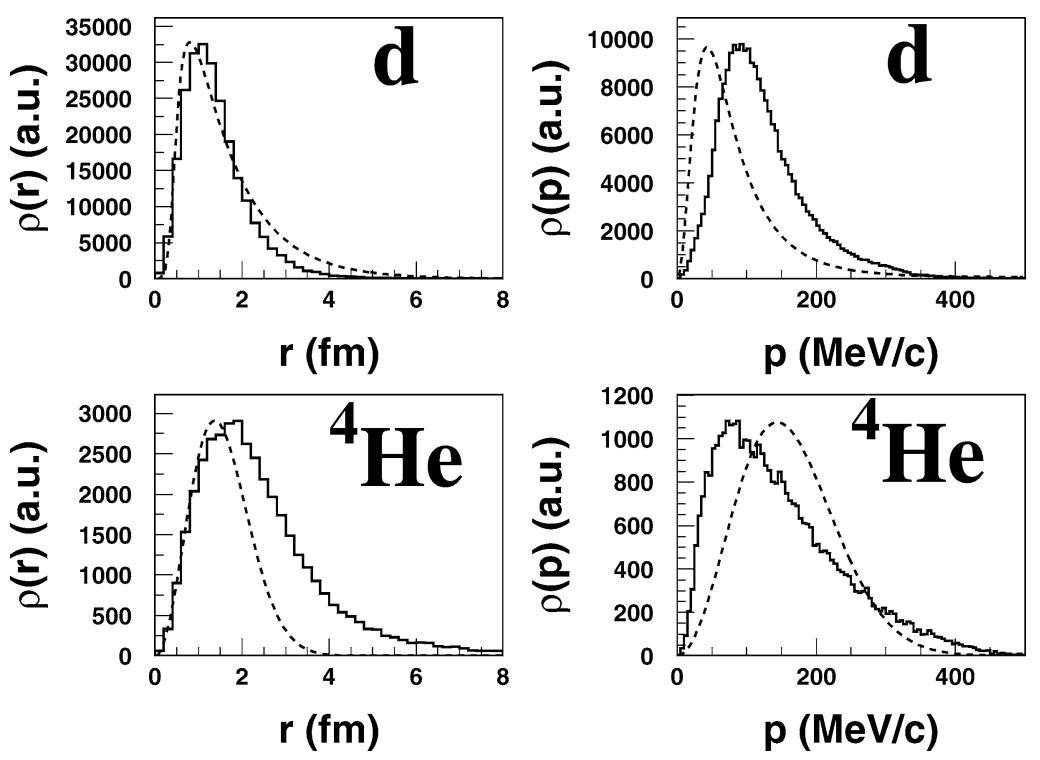

Fig. 6. Distributions (in arbitrary units) of the radial distance separating the position of the nucleons from the center of mass (left panels) and of the momentum of the nucleons in the rest frame of the cluster (right panels) for the "pre-deuterons" (upper part) and "pre-alphas" (lower part) generated in our model (histograms) for the $p+\mathrm{Au}$ system at $2.5 \mathrm{GeV}$. The dashed lines give the same distributions for free clusters, as given by the Paris potential for the deuteron and by a Gaussian model for the alpha particle, normalised in the same way. See text for detail.

deuterons" are on the average slightly more compact in configurational space than free deuterons, but are noticeably more extended in momentum space. The trend is reversed for the "pre- $\alpha$-clusters". The difference between "pre-clusters" and free clusters arises for two reasons: (i) our compactness criterion Eq. (2) slightly differs from compactness in a free deuteron, which assumes proximity both in configuration and momentum spaces; (ii) the INC dynamics can favour some particular regions of the phase space defined by Eq. (2). Let us finally notice that, in order to form a deuteron, for instance, in reality, a neutron and a proton need primarily to be close to each other in phase space, but also need to experience some extra soft interaction (from other nucleons or from the mean field) which transforms them into an on-shell deuteron. The relative success of our model justifies a posteriori the non-obvious possibility of simulating soft interactions by a simple geometrical model.

\section{Conclusion}

We have presented a model for the production of light charged clusters in the cascade stage of spallation reactions. This model contains novel features. It assumes that clusters are "formed" when a nucleon in the cascade model is candidate for being emitted. It relies on the instantaneous microscopic phase space occupancy in the nuclear surface region, dynamically generated by the INC model itself. It is not an entirely microscopic model, 
as we introduced two parameters, the most important one, $h_{0}$, describing the closeness condition for two nucleons belonging to the same cluster, the other one originating from technical considerations dictated by our INC code, in which nucleons are checked for emission when they are in the outer fringes of the nucleus.

This method opens the possibility of having a unified INC + evaporation model handling cluster emission on the same footing as nucleon emission, filling a long-standing gap. Non-evaporative light cluster emission has often been handled by introducing a so-called pre-equilibrium stage between the cascade and the evaporation stages. In this stage, based ordinarily on exciton models, emission of clusters is usually treated on a phenomenological basis, just by attaching cluster emission probabilities, generally fitted to experiment, to exciton configurations. Our method has thus the double advantage of being more microscopically founded and of allowing the emission of composites at any time.

Our aim in this paper was to demonstrate the potentiality of the model. That is why we did not vary the parameters of the model. We think that these parameters can reasonably be changed with the target mass and the incident energy (or the excitation energy). A variation with the nature of the cluster is equally acceptable, if not perhaps physically mandatory. With the simple choice adopted here, we obtain a promising agreement with the data for three systems at different incident energies, and this for a wide range of cluster kinetic energy.

We have shown that the inclusion of cluster production enhances the total (free + bound) nucleon yield in the cascade stage, whereas the evaporation stage remains basically the same. This has however rather unexpected results: the free neutron yield is reduced, the free proton yield is diminished slightly more and the fragmentation mass spectrum is broader. The first and third effects are improving our previous results without clusters.

Besides the determination of optimal parameters, other points are worth to be to investigated, such as the production of heavier composites and the extension of the model to low incident energy. Also, alternative choices for the closeness criterion Eq. (2) and the origin of the "pre-cluster" sizes deserve some further study. We plan to tackle these points in a future work.

\section{References}

[1] W. Gudowski, Nucl. Phys. A 654 (1999) 436c.

[2] G.D. Westfall, et al., Phys. Rev. C 17 (1978) 1368.

[3] E.N. Vol'nin, et al., Sov. Phys. JETP Lett. 19 (1974) 357.

[4] V.I. Bogatin, et al., Nucl. Phys. A 260 (1976) 446.

[5] J. Franz, et al., Nucl. Phys. A 472 (1987) 733.

[6] J. Franz, et al., Nucl. Phys. A 510 (1990) 774.

[7] A. Letourneau, et al., Nucl. Phys. A 712 (2002) 133.

[8] A. Boudard, J. Cugnon, S. Leray, C. Volant, Phys. Rev. C 66 (2002) 044615.

[9] J.-J. Gaimard, K.-H. Schmidt, Nucl. Phys. A 531 (1991) 709.

[10] A.R. Junghans, M. de Jong, H.G. Clerc, A.V. Ignatyuk, G.A. Kudyaev, K.-H. Schmidt, Nucl. Phys. A 629 (1998) 635.

[11] S.F. Butler, C.A. Pearson, Phys. Rev. 129 (1963) 836.

[12] A. Schwarzschild, Č. Zupančič, Phys. Rev. 129 (1963) 854.

[13] J. Cugnon, C. Volant, Z. Phys. A 334 (1989) 435. 
[14] K.K. Gudima, S.G. Mashnik, V.D. Toneev, Nucl. Phys. A 401 (1983) 329.

[15] S.G. Mashnik, A.J. Sierk, J. Nucl. Sci. Technol., Suppl. 2 (2002) 758, Los Alamos preprint LANL Report LA-UR-01-5390.

[16] J. Aichelin, Phys. Rep. 202 (1991) 233.

[17] G.F. Bertsch, H. Kruse, S. Das Gupta, Phys. Rev. C 29 (1984) 673.

[18] S. Ayik, Ch. Grégoire, Nucl. Phys. A 513 (1990) 187.

[19] D.H.E. Gross, Rep. Prog. Phys. 53 (1990) 605.

[20] J.P. Bondorf, R. Donangelo, I.N. Mishustin, C.J. Pethick, H. Schulz, K. Sneppen, Nucl. Phys. A 443 (1985) 321.

[21] H. Feldmeier, Nucl. Phys. A 515 (1990) 147.

[22] A. Ono, H. Horiuchi, Phys. Rev. C 51 (1995) 299.

[23] J. Cugnon, Nucl. Phys. A 462 (1987) 751.

[24] A. Lejeune, P. Grangé, M. Martzolff, J. Cugnon, Nucl. Phys. A 453 (1986) 189.

[25] J. Knoll, L. Münchow, G. Röpke, H. Schulz, Phys. Lett. B 112 (1982) 13.

[26] M. Baldo, U. Lombardo, P. Schuck, Phys. Rev. C 52 (1995) 975.

[27] A. Letourneau, Ph.D. Thesis, University of Caen, 2000.

[28] S. Furihata, Nucl. Instrum. Methods Phys. Res. B 171 (2000) 251;

S. Furihata, T. Nakamura, J. Nucl. Sci. Technol., Suppl. 2 (2002) 720, Tsukuba, Japan.

[29] S. Nagamyia, M. Gyulassy, Adv. Nucl. Phys. 13 (1984) 201.

[30] H.H. Gutbrod, et al., Phys. Rev. Lett. 37 (1976) 667.

[31] S. Leray, et al., Phys. Rev. C 65 (2002) 044621.

[32] M. Enke, et al., Nucl. Phys. A 657 (1997) 317.

[33] T. Enqvist, et al., Nucl. Phys. A 686 (2001) 481.

[34] M. Lacombe, B. Loiseau, J.-M. Richard, R. Vinh Mau, J. Coté, P. Pirès, R. de Tourreil, Phys. Rev. C 21 (1980) 861. 